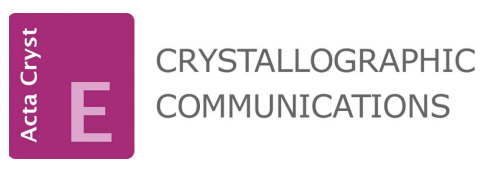

ISSN 2056-9890

Received 24 May 2019

Accepted 30 January 2020

Edited by C. Massera, Università di Parma, Italy

Keywords: crystal structure; nickel complexes; tren; tripodal ligand; hydrogen bonding.

CCDC reference: 1911584

Supporting information: this article has supporting information at journals.iucr.org/e

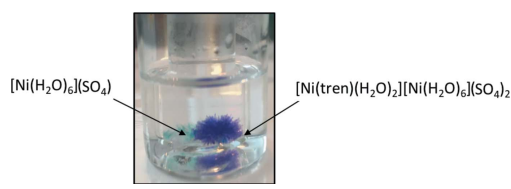

\section{Crystal structure of a nickel compound comprising two nickel(II) complexes with different ligand environments: $\left[\mathrm{Ni}(\right.$ tren $\left.)\left(\mathrm{H}_{2} \mathrm{O}\right)_{2}\right]\left[\mathrm{Ni}\left(\mathrm{H}_{2} \mathrm{O}\right)_{6}\right]\left(\mathrm{SO}_{4}\right)_{2}$}

\author{
Karilys González Nieves ${ }^{a *}$ and Dalice M. Piñero Cruz ${ }^{b}$
}

\begin{abstract}
${ }^{a}$ Department of Natural Sciences, University of Puerto Rico, Carolina Campus, 2100 Avenida Sur, Carolina, PR 00987, Puerto Rico, and ${ }^{\mathbf{b}}$ Department of Chemistry, University of Puerto Rico, Rio Piedras Campus, Ponce de Leon Avenue, San Juan, PR 00931, Puerto Rico. *Correspondence e-mail: karilys.gonzalez@upr.edu
\end{abstract}

The title compound, diaqua[tris(2-aminoethyl)amine]nickel(II) hexaaquanickel(II) bis(sulfate), $\left[\mathrm{Ni}\left(\mathrm{C}_{6} \mathrm{H}_{18} \mathrm{~N}_{4}\right)\left(\mathrm{H}_{2} \mathrm{O}\right)_{2}\right]\left[\mathrm{Ni}\left(\mathrm{H}_{2} \mathrm{O}\right)_{6}\right]\left(\mathrm{SO}_{4}\right)_{2}$ or [Ni(tren)$\left.\left(\mathrm{H}_{2} \mathrm{O}\right)_{2}\right]\left[\mathrm{Ni}\left(\mathrm{H}_{2} \mathrm{O}\right)_{6}\right]\left(\mathrm{SO}_{4}\right)_{2}$, consists of two octahedral nickel complexes within the same unit cell. These metal complexes are formed from the reaction of $\left[\mathrm{Ni}\left(\mathrm{H}_{2} \mathrm{O}\right)_{6}\right]\left(\mathrm{SO}_{4}\right)$ and the ligand tris(2-aminoethyl)amine (tren). The crystals of the title compound are purple, different from those of the starting complex $\left[\mathrm{Ni}\left(\mathrm{H}_{2} \mathrm{O}\right)_{6}\right]\left(\mathrm{SO}_{4}\right)$, which are turquoise. The reaction was performed both in a 1:1 and 1:2 metal-ligand molar ratio, always yielding the co-precipitation of the two types of crystals. The asymmetric unit of the title compound, which crystallizes in the space group Pnma, consists of two half $\mathrm{Ni}^{\mathrm{iI}}$ complexes and a sulfate counteranion. The mononuclear cationic complex $\left[\mathrm{Ni}(\operatorname{tren})\left(\mathrm{H}_{2} \mathrm{O}\right)_{2}\right]^{2+}$ comprises an $\mathrm{Ni}$ ion, the tren ligand and two water molecules, while the mononuclear complex $\left[\mathrm{Ni}\left(\mathrm{H}_{2} \mathrm{O}\right)_{6}\right]^{2+}$ consists of another $\mathrm{Ni}$ ion surrounded by six coordinated water molecules. The $\left[\mathrm{Ni}(\operatorname{tren})\left(\mathrm{H}_{2} \mathrm{O}\right)_{2}\right]$ and $\left[\mathrm{Ni}\left(\mathrm{H}_{2} \mathrm{O}\right)_{6}\right]$ subunits are connected to the $\mathrm{SO}_{4}{ }^{2-}$ counter-anions through hydrogen bonding, thus consolidating the crystal structure.

\section{Chemical context}

Tris(2-aminoethyl)amine (tren) has been used extensively as an ancillary tripodal ligand for capping transition metals to form mononuclear and polynuclear complexes. The tren ligand has the capacity to chelate metal ions through its central tertiary amine and through its three terminal amine groups in a spider-like conformation, leaving one or two positions available for additional ligand coordination (Marzotto et al., 1993; Albertin et al., 1975; Blackman, 2005; Brines et al., 2007). Metal complexes with a variety of ligands in which also tren is coordinating to the metal center have been proposed for applications in catalysis (Ruffin et al., 2017), sensors, and as precursors of bioinorganic reactions (Sakai et al., 1996). For instance, $\mathrm{Ni}($ tren) complexes have been proposed for applications in biological systems (Salam \& Aoki, 2001) or as a model to study enantioselective synthesis or asymmetric catalysis (Rao et al., 2009), and as coordination polymers in magnetism, electrical conductivity and ion exchange (Park et al., 2001; Tanase et al., 1996). $\left[\mathrm{Ni}(\operatorname{tren})\left(\mathrm{H}_{2} \mathrm{O}\right)_{2}\right]$ was reported previously (Chen et al., 2001; Pedersen et al., 2014); however, to our knowledge, this is the first report of it co-crystallizing with the hexaaquo nickel complex $\left[\mathrm{Ni}\left(\mathrm{H}_{2} \mathrm{O}\right)_{6}\right]\left(\mathrm{SO}_{4}\right)$. 

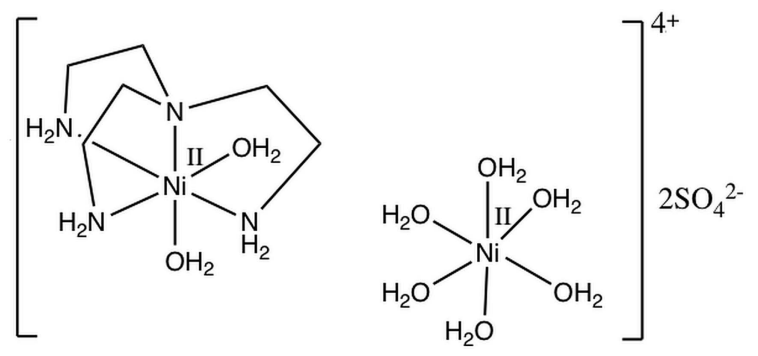

\section{Structural commentary}

Fig. 1 shows the molecular structure of the title compound, which crystallizes in the space group Pnma. Its asymmetric unit comprises two half $\mathrm{Ni}^{\mathrm{II}}$ complexes and a sulfate counteranion. Each Ni complex shows a different ligand environment: (i) the mononuclear cationic complex $\left[\mathrm{Ni}(\operatorname{tren})\left(\mathrm{H}_{2} \mathrm{O}\right)_{2}\right]^{2+}$ includes Ni1, the tren ligand and two water molecules; (ii) the mononuclear complex $\left[\mathrm{Ni}\left(\mathrm{H}_{2} \mathrm{O}\right)_{6}\right]^{2+}$ consists of $\mathrm{Ni} 2$ surrounded by six coordinated water molecules.

Ni1 exhibits an octahedral geometry of the type $\mathrm{N}_{4} \mathrm{O}_{2}$, with the central $\mathrm{N} 1$ atom of the tren ligand occupying one of the axial positions and atoms $\mathrm{N} 2, \mathrm{~N} 3$ and $\mathrm{N} 2{ }^{\mathrm{i}}$ occupying three of the equatorial positions [symmetry code: (i) $x,-y+\frac{1}{2}, z$ ]. The remaining two positions, one axial $(\mathrm{O} 2)$ and one equatorial (O1), are occupied by two oxygen atoms from the two water molecules. The bond lengths are similar for the Ni1 $-\mathrm{N}$ bonds that are trans to oxygen atoms; for instance, $\mathrm{Ni} 1-\mathrm{N} 1_{\mathrm{ax}}$ is 2.064 (2) $\AA$ and $\mathrm{Ni} 1-\mathrm{N}_{\text {eq }}$ is $2.069(2) \AA$; a longer bond distance is observed between Ni1- $2_{2}$ eq 2.122 (2), which is trans by symmetry to another nitrogen atom, $\mathrm{N} 2^{\mathrm{i}}$. The nickeloxygen bond length is shorter for Ni1 $-\mathrm{O} 2_{\mathrm{ax}}$ at 2.094 (2) $\AA$, in comparison to $\mathrm{Ni1}-\mathrm{O} 1_{\mathrm{eq}}$, which is 2.140 (2) $\AA$. The N3 and $\mathrm{C} 3$ atoms of the tren ligand lie on a mirror plane perpendicular to [010]. This results in a symmetry-induced disorder of the $\mathrm{N} 3 / \mathrm{C} 4 / \mathrm{C} 3$ fragment. The octahedral geometry around the Ni1 ion is reflected by the angles $\mathrm{N} 1-\mathrm{Ni} 1-\mathrm{O} 2=178.42(8)^{\circ}$, $\mathrm{N} 2-\mathrm{Ni} 1-\mathrm{N} 2^{\mathrm{i}}=164.74(9)^{\circ}$, and $\mathrm{N} 3-\mathrm{Ni} 1-\mathrm{O} 1=177.27(8)^{\circ}$.
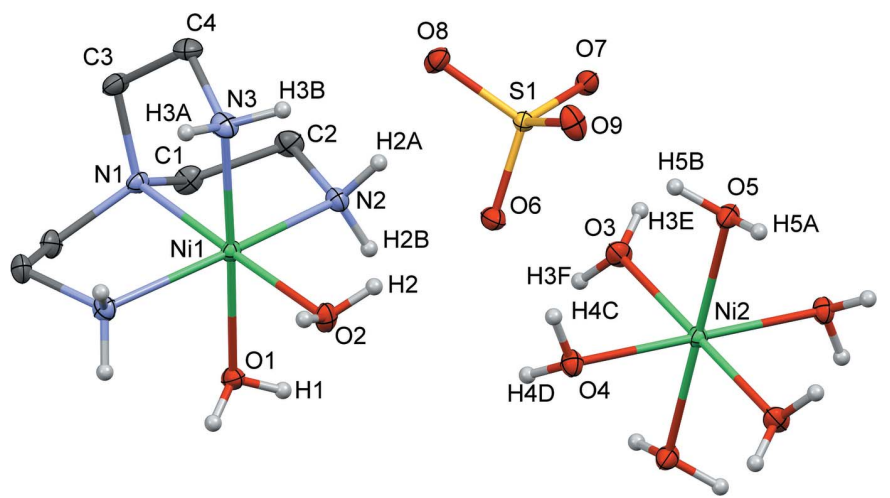

Figure 1

View of the molecular structure of the title compound with displacement ellipsoids drawn at the $20 \%$ probability level and labeling scheme for the symmetry-independent atoms. The $\mathrm{CH}_{2}$ hydrogen atoms have been omitted for clarity. The symmetry operations generating the equivalent atoms are $1-x, 1-y, 2-z$ and $x, \frac{1}{2}-y, z$ for $\left.\left[\mathrm{Ni}^{(} \mathrm{H}_{2} \mathrm{O}\right)_{6}\right]^{2+}$ and $\left[\mathrm{Ni}(\operatorname{tren})\left(\mathrm{H}_{2} \mathrm{O}\right)_{2}\right]^{2+}$, respectively.
Table 1

Hydrogen-bond geometry $\left(\AA,^{\circ}\right)$.

\begin{tabular}{|c|c|c|c|c|}
\hline$D-\mathrm{H} \cdots A$ & $D-\mathrm{H}$ & $\mathrm{H} \cdots A$ & $D \cdots A$ & $D-\mathrm{H} \cdots A$ \\
\hline $\mathrm{O} 1-\mathrm{H} 1 \cdots \mathrm{O}^{\mathrm{i}}$ & $0.78(2)$ & $2.05(2)$ & $2.8212(16)$ & $172(2)$ \\
\hline $\mathrm{O} 2-\mathrm{H} 2 \cdots \mathrm{O} 6$ & $0.81(2)$ & $1.96(2)$ & $2.7342(15)$ & $162(3)$ \\
\hline $\mathrm{O} 3-\mathrm{H} 3 E \cdots \mathrm{O} 9^{\mathrm{ii}}$ & $0.81(2)$ & $1.94(2)$ & $2.731(2)$ & $167(2)$ \\
\hline $\mathrm{O} 3-\mathrm{H} 3 F \cdots \mathrm{O}^{\mathrm{i}}$ & $0.85(2)$ & $2.05(2)$ & $2.8403(18)$ & $155(2)$ \\
\hline $\mathrm{O} 4-\mathrm{H} 4 C \cdots \mathrm{O} 6$ & $0.83(2)$ & $1.91(2)$ & $2.7249(18)$ & $171(2)$ \\
\hline $\mathrm{O} 4-\mathrm{H} 4 D \cdots \mathrm{O} 8^{\mathrm{i}}$ & $0.83(2)$ & $1.95(2)$ & $2.7810(18)$ & $179(2)$ \\
\hline $\mathrm{O} 5-\mathrm{H} 5 A \cdots \mathrm{O} 7^{\mathrm{iii}}$ & 0.88 & 2.02 & $2.8125(19)$ & 150 \\
\hline $\mathrm{O} 5-\mathrm{H} 5 B \cdots \mathrm{O} 7$ & 0.88 & 1.95 & $2.7826(17)$ & 160 \\
\hline
\end{tabular}

The Ni2 ion of the mononuclear complex $\left[\mathrm{Ni}\left(\mathrm{H}_{2} \mathrm{O}\right)_{6}\right]^{2+}$ also shows an octahedral geometry. In the asymmetric unit, the atom $\mathrm{Ni} 2$ sits on an inversion center on a screw axis along the $b$-axis direction. The $\mathrm{Ni} 2-\mathrm{O}_{\text {water }}$ bond lengths with $\mathrm{O} 3, \mathrm{O} 4$ and O5 range between 2.051 (1) and 2.074 (1) $\AA$, respectively, with angles of $180^{\circ}$ due to symmetry.

\section{Supramolecular features}

The crystal structure of the title compound is consolidated through intermolecular hydrogen bonding between the water molecules from the $\left[\mathrm{Ni}(\operatorname{tren})\left(\mathrm{H}_{2} \mathrm{O}\right)_{2}\right]$ complex, the sulfate oxygen atoms and the water molecules from the $\left[\mathrm{Ni}\left(\mathrm{H}_{2} \mathrm{O}\right)_{6}\right]$ complex (Fig. 2 and Table 1). In particular, the two water molecules of $\left[\mathrm{Ni}(\operatorname{tren})\left(\mathrm{H}_{2} \mathrm{O}\right)_{2}\right]$ form $\mathrm{O} 1-\mathrm{H} 1 \cdots \mathrm{O} 8^{\mathrm{i}}$ and $\mathrm{O} 2-$ $\mathrm{H} 2$. . O6 hydrogen bonds of 2.05 (2) and 1.96 (2) $\AA$ respectively, involving two neighboring $\mathrm{SO}_{4}{ }^{2-}$ anions [symmetry code: (i) $\left.x+\frac{1}{2}, y,-z+\frac{3}{2}\right)$. The $\left[\mathrm{Ni}\left(\mathrm{H}_{2} \mathrm{O}\right)_{6}\right]$ complex is hydrogen bonded to adjacent $\mathrm{SO}_{4}{ }^{2-}$ anions through $\mathrm{O} 3-\mathrm{H} 3 E \cdots \mathrm{O} 9^{\mathrm{ii}}$, $\mathrm{O} 3-\mathrm{H} 3 F \cdots \mathrm{O}^{\mathrm{i}}, \quad \mathrm{O} 4-\mathrm{H} 4 C \cdots \mathrm{O} 6, \quad \mathrm{O} 4-\mathrm{H} 4 D \cdots \mathrm{O} 8^{\mathrm{i}}, \mathrm{O} 5-$ $\mathrm{H} 5 B \cdots \mathrm{O} 7, \mathrm{O} 5-\mathrm{H} 5 A \cdots \mathrm{O} 7^{\mathrm{iii}}$ contacts [symmetry codes: (ii) $-x+\frac{1}{2},-y+1, z-\frac{1}{2}$; (iii) $\left.-x+\frac{1}{2},-y+1, z+\frac{1}{2}\right]$. These hydrogen-bond distances range from 1.905 (15) to 2.047 (18) A. Additional weak hydrogen bonds are formed between the hydrogen atoms from the primary amine groups of the tren ligand and the sulfate oxygen atoms.

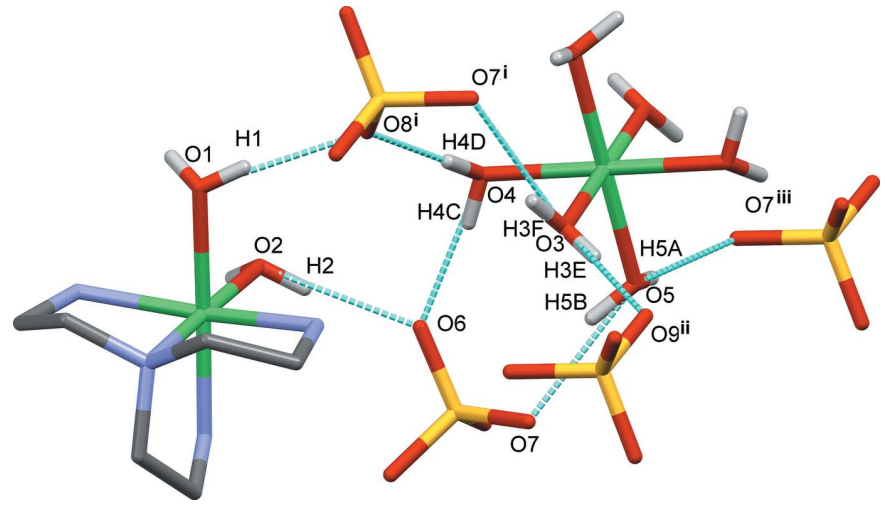

Figure 2

The hydrogen-bonding network (cyan dotted lines) in the title compound. Symmetry codes: (i) $x+\frac{1}{2}, y,-z+\frac{3}{2}$; (ii) $-x+\frac{1}{2},-y+1, z-\frac{1}{2}$; (iii) $-x+\frac{1}{2}$, $-y+1, z+\frac{1}{2}$. 


\section{Database survey}

A search for tris(2-aminoethyl)aminenickel complexes in the Cambridge Structural Database (CSD version 5.38, updated February 2019; Groom et al., 2016) yielded 222 hits. Among these results, 124 hits contained the ligand tris(2-aminoethyl)amine capping the nickel ion, along with other types of ligands on the remaining coordination sites. Only two hits contain the diaqua[tris(2-aminoethyl)amine]nickel(II) complex, [ $\mathrm{Ni}(\operatorname{tren})\left(\mathrm{H}_{2} \mathrm{O}\right)_{2}$ ] (LUMVIY; Chen et al., 2001; TIYQAT; Tanase et al., 1996). More precisely, the asymmetric unit in LUMVIY comprises the $\left[\mathrm{Ni}(\operatorname{tren})\left(\mathrm{H}_{2} \mathrm{O}\right)_{2}\right]^{2+}$ cation with two independent halves of a 1,5-naphthalenedisulfonate (1,5nds) ligand as counter-anion. A common feature of this structure with the title compound is the hydrogen bond network formed between the water molecules on the Ni(tren) motif with the counter anions. However, in the title compound, also the hydrogen atoms on the primary amine groups form hydrogen bonds with the sulfate anions, albeit quite weak. In TIYQAT, sulfate anions act as counter-ions for the $\left[\mathrm{Ni}(\operatorname{tren})\left(\mathrm{H}_{2} \mathrm{O}\right)_{2}\right]^{2+}$ complex, and uncoordinated water molecules are included in the crystal lattice. The angle between the $\mathrm{Ni}$ center and the two oxygen atoms from the coordinated water molecules are $86.52(5)^{\circ}(\mathrm{O} 7-\mathrm{Ni1}-\mathrm{O} 8)$ and $86.9(4)^{\circ}(\mathrm{O} 5-\mathrm{Ni1}-\mathrm{O} 6)$ for LUMVIY and TIYQAT, respectively. The corresponding angle $\mathrm{O} 2-\mathrm{Ni}-\mathrm{O} 1$ in the tittle compound has a value of $88.70(8)^{\circ}$, which is in good agreement with the reported values. The title compound is the first example of a crystal structure of $\left[\mathrm{Ni}(\operatorname{tren})\left(\mathrm{H}_{2} \mathrm{O}\right)_{2}\right]^{2+}$ cocrystallizing with the $\left[\mathrm{Ni}\left(\mathrm{H}_{2} \mathrm{O}\right)_{6}\right]^{2+}$ complex.

\section{Synthesis and crystallization}

The synthesis of the title compound is summarized in the reaction scheme shown in Fig. 3. $\mathrm{NiSO}_{4} \cdot 6 \mathrm{H}_{2} \mathrm{O}$ and tris $(2-$ aminoethyl)amine (tren) were used without further purification. A methanolic solution of $\mathrm{NiSO}_{4} \cdot 6 \mathrm{H}_{2} \mathrm{O}(0.0265 \mathrm{~g}$, $0.1 \mathrm{mmol})$ was added slowly to a tren $(0.0146 \mathrm{~g}, 0.1 \mathrm{mmol})$ solution $(4 \mathrm{~mL} \mathrm{MeOH})$ at room temperature. The resulting solution was stirred for two $\mathrm{h}$ and it changed color from light green to purple. The solution was then filtered through celite and evaporated under reduced pressure. Single crystals of the title compound were obtained by vapor diffusion of methanol into 2-propanol. In the crystallization process, two types of crystal were formed: the starting reagent hexahydrate nickel (II) complex (turquoise crystals) and the nickel(II) tren complex (purple crystals, Fig. 4). The reaction was performed both in a 1:1 and 1:2 metal-ligand molar ratio, always yielding

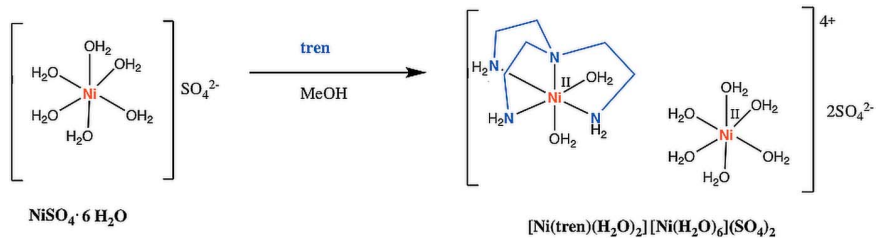

Figure 3

Reaction scheme for the synthesis of $\left[\mathrm{Ni}(\operatorname{tren})\left(\mathrm{H}_{2} \mathrm{O}\right)_{2}\right]\left[\mathrm{Ni}\left(\mathrm{H}_{2} \mathrm{O}\right)_{6}\right]\left(\mathrm{SO}_{4}\right)_{2}$.
Table 2

Experimental details.

\begin{tabular}{|c|c|}
\hline \multicolumn{2}{|l|}{ Crystal data } \\
\hline Chemical formula & $\begin{array}{l}{\left[\mathrm{Ni}\left(\mathrm{C}_{6} \mathrm{H}_{18} \mathrm{~N}_{4}\right)\left(\mathrm{H}_{2} \mathrm{O}\right)_{2}\right][\mathrm{Ni}(-} \\
\left.\left.\quad \mathrm{H}_{2} \mathrm{O}\right)_{6}\right]\left(\mathrm{SO}_{4}\right)_{2}\end{array}$ \\
\hline$M_{\mathrm{r}}$ & 599.91 \\
\hline Crystal system, space group & Orthorhombic, Pnma \\
\hline Temperature $(\mathrm{K})$ & 293 \\
\hline$a, b, c(\AA)$ & $11.8937(1), 21.3933(2), 8.4468(1)$ \\
\hline$V\left(\AA^{3}\right)$ & $2149.25(4)$ \\
\hline$Z$ & 4 \\
\hline Radiation type & $\mathrm{Cu} K \alpha$ \\
\hline$\mu\left(\mathrm{mm}^{-1}\right)$ & 4.76 \\
\hline Crystal size $(\mathrm{mm})$ & $0.28 \times 0.21 \times 0.09$ \\
\hline \multicolumn{2}{|l|}{ Data collection } \\
\hline Diffractometer & $\begin{array}{l}\text { Rigaku Oxford Diffraction Super- } \\
\text { Nova, Single source at offset/far, } \\
\text { HyPix3000 }\end{array}$ \\
\hline Absorption correction & $\begin{array}{l}\text { Multi-scan (CrysAlis PRO; Rigaku } \\
\text { OD, 2015) }\end{array}$ \\
\hline$T_{\min }, T_{\max }$ & $0.353,0.661$ \\
\hline $\begin{array}{l}\text { No. of measured, independent and } \\
\text { observed }[I>2 \sigma(I)] \text { reflections }\end{array}$ & $17858,2044,1996$ \\
\hline$R_{\text {int }}$ & 0.023 \\
\hline$(\sin \theta / \lambda)_{\max }\left(\AA^{-1}\right)$ & 0.605 \\
\hline \multicolumn{2}{|l|}{ Refinement } \\
\hline$R\left[F^{2}>2 \sigma\left(F^{2}\right)\right], w R\left(F^{2}\right), S$ & $0.023,0.063,1.12$ \\
\hline No. of reflections & 2044 \\
\hline No. of parameters & 173 \\
\hline No. of restraints & 8 \\
\hline $\mathrm{H}$-atom treatment & All $\mathrm{H}$-atom parameters refined \\
\hline$\Delta \rho_{\max }, \Delta \rho_{\min }\left(\mathrm{e} \AA^{-3}\right)$ & $0.37,-0.35$ \\
\hline
\end{tabular}

Computer programs: CrysAlis PRO (Rigaku OD, 2015), olex2.solve (Bourhis et al., 2015), SHELXL2016 (Sheldrick, 2015) and OLEX2 (Dolomanov et al., 2009).

the title compound. IR data: $3265(m), 3171(m), 2937(w)$, $2891(w), 1607(m), 1472(w) 1338(w), 1054(s), 984(m), 885$ $(m), 750(w), 685(w)$.

\section{Refinement}

Crystal data, data collection and structure refinement details are summarized in Table 2. $\mathrm{H}$ atoms were included in geometrically calculated positions for the alkyl and amine groups using a riding model: $\mathrm{C}-\mathrm{H}=0.97 \AA$ and $\mathrm{N}-\mathrm{H}=$ $0.89 \AA$ with $U_{\text {iso }}(\mathrm{H})=1.2 U_{\text {eq }}(\mathrm{C}, \mathrm{N})$. The hydrogen atoms of the water molecules were located from the difference-Fourier map; they were refined freely in the case of $\mathrm{O} 1$ and $\mathrm{O} 2$, with a DFIX of $0.85(2) \AA$ and $U_{\text {iso }}(\mathrm{H})=1.5 U_{\text {eq }}(\mathrm{O})$ in the case of $\mathrm{O} 3$ and $\mathrm{O} 4$, and riding with $\mathrm{O}-\mathrm{H}=0.88 \AA$ and $U_{\text {iso }}(\mathrm{H})$ $=1.5 U_{\text {eq }}(\mathrm{O})$ in the case of $\mathrm{O} 5$.

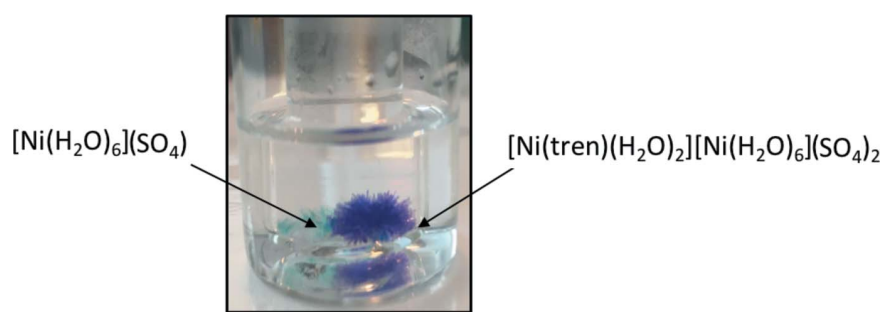

Figure 4

Crystallization of $\left[\mathrm{Ni}(\right.$ tren $\left.)\left(\mathrm{H}_{2} \mathrm{O}\right)_{2}\right]\left[\mathrm{Ni}\left(\mathrm{H}_{2} \mathrm{O}\right)_{6}\right]\left(\mathrm{SO}_{4}\right)_{2}$ and $\left[\mathrm{Ni}\left(\mathrm{H}_{2} \mathrm{O}\right)_{6}\right] \mathrm{SO}_{4}$ in the same reaction vial. 
The N3 and C3 atoms of the tren ligand lie on a mirror plane perpendicular to [010]. This results in a symmetry-induced disorder of the N3/C4/C3 fragment.

\section{Acknowledgements}

We are grateful to the Department of Natural Science at UPR Carolina Campus (Department of Education, grant No. PO31S130068; however, those contents do not necessarily represent the policy of the Department of Education, and you should not assume endorsement by the Federal Government) and the University of Puerto Rico's Molecular Sciences Research Center for the use of the Rigaku XTLab SuperNova diffractometer. Special thanks to Dr Indranil Chakraborty for consultation on the final refinement of the structure.

\section{Funding information}

This material is based upon work supported by the National Science Foundation under grant No. 1626103. This study was supported by an Institutional Development Award (IDeA) INBRE grant No. P20GM103475 from the National Institute of General Medical Sciences (NIGMS), a component of the National Institutes of Health (NIH), and the Bioinformatics Research Core of the INBRE. Its contents are solely the responsibility of the authors and do not necessarily represent the official view of NIGMS or NIH.

\section{References}

Albertin, G., Bordignon, E. \& Orio, A. A. (1975). Inorg. Chem. 14, 1411-1413.

Blackman, A. G. (2005). Polyhedron, 24, 1-39.

Bourhis, L. J., Dolomanov, O. V., Gildea, R. J., Howard, J. A. K. \& Puschmann, H. (2015). Acta Cryst. A71, 59-75.

Brines, L. M., Shearer, J., Fender, J. K., Schweitzer, D., Shoner, S. C., Barnhart, D., Kaminsky, W., Lovell, S. \& Kovacs, J. A. (2007). Inorg. Chem. 46, 9267-9277.

Chen, C., Cai, J., Feng, X. \& Chen, X. (2001). J. Chem. Crystallogr. 31, 271-280.

Dolomanov, O. V., Bourhis, L. J., Gildea, R. J., Howard, J. A. K. \& Puschmann, H. (2009). J. Appl. Cryst. 42, 339-341.

Groom, C. R., Bruno, I. J., Lightfoot, M. P. \& Ward, S. C. (2016). Acta Cryst. B72, 171-179.

Marzotto, A., Clemente, D. A., Ciccarese, A. \& Valle, G. (1993). J. Crystallogr. Spectrosc. Res. 23, 119-131.

Park, H. W., Sung, S. M., Min, K. S., Bang, H. \& Suh, M. P. (2001). Eur. J. Inorg. Chem. pp. 2857-2863.

Pedersen, K. S., Bendix, J. \& Clérac, R. (2014). Chem. Commun. 50, 4396-4415.

Rao, S. A., Pal, A., Ghosh, R. \& Das, S. K. (2009). Inorg. Chem. 48, 10476.

Rigaku OD (2015). CrysAlis PRO. Rigaku Oxford Diffraction, Yarnton, England.

Ruffin, H., Boussambe, G. N. M., Roisnel, T., Dorcet, V., Boitrel, B. \& Le Gac, S. (2017). J. Am. Chem. Soc. 139, 13847-13857.

Sakai, K., Yamada, Y. \& Tsubomura, T. (1996). Inorg. Chem. 35, 3163-3172.

Salam, A. Md. \& Aoki, K. (2001). Inorg. Chim. Acta, 314, 71-82.

Sheldrick, G. M. (2015). Acta Cryst. C71, 3-8.

Tanase, T., Doi, M., Nouchi, R., Kato, M., Sato, Y., Ishida, K., Kobayashi, K., Sakurai, T., Yamamoto, Y. \& Yano, S. (1996). Inorg. Chem. 35, 4848-4857. 


\section{supporting information}

Acta Cryst. (2020). E76, 314-317 [https://doi.org/10.1107/S2056989020001358]

\section{Crystal structure of a nickel compound comprising two nickel(II) complexes with different ligand environments: $\left[\mathrm{Ni}(\operatorname{tren})\left(\mathrm{H}_{2} \mathrm{O}\right)_{2}\right]\left[\mathrm{Ni}\left(\mathrm{H}_{2} \mathrm{O}\right)_{6}\right]\left(\mathrm{SO}_{4}\right)_{2}$}

\section{Karilys González Nieves and Dalice M. Piñero Cruz}

Computing details

Data collection: CrysAlis PRO (Rigaku OD, 2015); cell refinement: CrysAlis PRO (Rigaku OD, 2015); data reduction: CrysAlis PRO (Rigaku OD, 2015); program(s) used to solve structure: olex2.solve (Bourhis et al., 2015); program(s) used to refine structure: SHELXL2016 (Sheldrick, 20156); molecular graphics: OLEX2 (Dolomanov et al., 2009); software used to prepare material for publication: OLEX2 (Dolomanov et al., 2009).

Diaqua[tris(2-aminoethyl)amine]nickel(II) hexaaquanickel(II) bis(sulfate)

Crystal data

$\left[\mathrm{Ni}\left(\mathrm{C}_{6} \mathrm{H}_{18} \mathrm{~N}_{4}\right)\left(\mathrm{H}_{2} \mathrm{O}\right)_{2}\right]\left[\mathrm{Ni}\left(\mathrm{H}_{2} \mathrm{O}\right)_{6}\right]\left(\mathrm{SO}_{4}\right)_{2}$

$M_{r}=599.91$

Orthorhombic, Pnma

$a=11.8937(1) \AA$

$b=21.3933(2) \AA$

$c=8.4468(1) \AA$

$V=2149.25(4) \AA^{3}$

$Z=4$

$F(000)=1256$

Data collection

Rigaku Oxford Diffraction SuperNova, Single source at offset/far, HyPix 3000 diffractometer

$\omega$ scans

Absorption correction: multi-scan

(CrysAlis PRO; Rigaku OD, 2015)

$T_{\min }=0.353, T_{\max }=0.661$

17858 measured reflections

Refinement

Refinement on $F^{2}$

Least-squares matrix: full

$R\left[F^{2}>2 \sigma\left(F^{2}\right)\right]=0.023$

$w R\left(F^{2}\right)=0.063$

$S=1.12$

2044 reflections

173 parameters

8 restraints

Primary atom site location: dual

Secondary atom site location: difference Fourier map
$D_{\mathrm{x}}=1.854 \mathrm{Mg} \mathrm{m}^{-3}$

$\mathrm{Cu} K \alpha$ radiation, $\lambda=1.54184 \AA$

Cell parameters from 14387 reflections

$\theta=3.7-68.8^{\circ}$

$\mu=4.76 \mathrm{~mm}^{-1}$

$T=293 \mathrm{~K}$

Block, clear violet

$0.28 \times 0.21 \times 0.09 \mathrm{~mm}$

2044 independent reflections

1996 reflections with $I>2 \sigma(I)$

$R_{\text {int }}=0.023$

$\theta_{\text {max }}=68.9^{\circ}, \theta_{\min }=4.1^{\circ}$

$h=-14 \rightarrow 14$

$k=-25 \rightarrow 25$

$l=-10 \rightarrow 10$

Hydrogen site location: mixed

All $\mathrm{H}$-atom parameters refined

$w=1 /\left[\sigma^{2}\left(F_{\mathrm{o}}^{2}\right)+(0.0312 P)^{2}+1.2986 P\right]$

where $P=\left(F_{\mathrm{o}}^{2}+2 F_{\mathrm{c}}{ }^{2}\right) / 3$

$(\Delta / \sigma)_{\max }<0.001$

$\Delta \rho_{\max }=0.37 \mathrm{e} \AA^{-3}$

$\Delta \rho_{\min }=-0.35$ e $\AA^{-3}$

Extinction correction: SHELXL2016

(Sheldrick, 2015),

$\mathrm{Fc}^{*}=\mathrm{kFc}\left[1+0.001 \mathrm{xFc}^{2} \lambda^{3} / \sin (2 \theta)\right]^{-1 / 4}$

Extinction coefficient: 0.00044 (5) 


\section{Special details}

Geometry. All esds (except the esd in the dihedral angle between two 1.s. planes) are estimated using the full covariance matrix. The cell esds are taken into account individually in the estimation of esds in distances, angles and torsion angles; correlations between esds in cell parameters are only used when they are defined by crystal symmetry. An approximate (isotropic) treatment of cell esds is used for estimating esds involving l.s. planes.

Fractional atomic coordinates and isotropic or equivalent isotropic displacement parameters $\left(\AA^{2}\right)$

\begin{tabular}{|c|c|c|c|c|c|}
\hline & $x$ & $y$ & $z$ & $U_{\text {iso }} * / U_{\text {eq }}$ & Occ. $(<1)$ \\
\hline Ni1 & $0.31517(3)$ & 0.250000 & $0.58049(4)$ & $0.01987(12)$ & \\
\hline $\mathrm{O} 1$ & $0.49430(15)$ & 0.250000 & $0.5573(2)$ & $0.0285(4)$ & \\
\hline H1 & $0.523(2)$ & $0.2795(11)$ & $0.592(3)$ & $0.042(7)^{*}$ & \\
\hline $\mathrm{O} 2$ & $0.33527(17)$ & 0.250000 & $0.8268(2)$ & $0.0315(4)$ & \\
\hline $\mathrm{H} 2$ & $0.304(2)$ & $0.2795(11)$ & $0.867(3)$ & $0.056(8)^{*}$ & \\
\hline N1 & $0.29062(16)$ & 0.250000 & $0.3386(2)$ & $0.0237(4)$ & \\
\hline $\mathrm{N} 2$ & $0.32729(13)$ & $0.34830(7)$ & $0.55183(18)$ & $0.0291(3)$ & \\
\hline $\mathrm{H} 2 \mathrm{~A}$ & 0.275056 & 0.367313 & 0.609911 & $0.035^{*}$ & \\
\hline $\mathrm{H} 2 \mathrm{~B}$ & 0.394537 & 0.361563 & 0.583583 & $0.035^{*}$ & \\
\hline N3 & $0.14137(18)$ & 0.250000 & $0.5913(3)$ & $0.0330(5)$ & \\
\hline $\mathrm{H} 3 \mathrm{~A}$ & 0.116429 & 0.211740 & 0.613150 & $0.040^{*}$ & 0.5 \\
\hline $\mathrm{H} 3 \mathrm{~B}$ & 0.118127 & 0.275722 & 0.667351 & $0.040^{*}$ & 0.5 \\
\hline $\mathrm{C} 1$ & $0.34686(17)$ & $0.30780(9)$ & $0.2826(2)$ & $0.0336(4)$ & \\
\hline $\mathrm{H} 1 \mathrm{~A}$ & 0.327506 & 0.315269 & 0.172689 & $0.040^{*}$ & \\
\hline H1B & 0.427761 & 0.302752 & 0.289509 & $0.040^{*}$ & \\
\hline $\mathrm{C} 2$ & $0.31074(17)$ & $0.36314(9)$ & $0.3821(2)$ & $0.0362(4)$ & \\
\hline $\mathrm{H} 2 \mathrm{C}$ & 0.354856 & 0.399608 & 0.353854 & $0.043^{*}$ & \\
\hline $\mathrm{H} 2 \mathrm{D}$ & 0.232177 & 0.372476 & 0.362353 & $0.043^{*}$ & \\
\hline $\mathrm{C} 3$ & $0.1684(2)$ & 0.250000 & $0.3008(3)$ & $0.0344(6)$ & \\
\hline $\mathrm{H} 3 \mathrm{C}$ & 0.145952 & 0.208172 & 0.269741 & $0.041^{*}$ & 0.5 \\
\hline $\mathrm{H} 3 \mathrm{D}$ & 0.154994 & 0.277594 & 0.211689 & $0.041^{*}$ & 0.5 \\
\hline $\mathrm{C} 4$ & $0.0975(3)$ & 0.27067 (19) & $0.4375(5)$ & $0.0384(10)$ & 0.5 \\
\hline $\mathrm{H} 4 \mathrm{~A}$ & 0.092804 & 0.315934 & 0.437065 & $0.046^{*}$ & 0.5 \\
\hline H4B & 0.022018 & 0.254369 & 0.424014 & $0.046^{*}$ & 0.5 \\
\hline $\mathrm{Ni} 2$ & 0.500000 & 0.500000 & 1.000000 & $0.02058(12)$ & \\
\hline $\mathrm{O} 3$ & $0.46413(12)$ & $0.51163(6)$ & $0.76229(15)$ & $0.0337(3)$ & \\
\hline $\mathrm{H} 3 \mathrm{E}$ & $0.4398(19)$ & $0.5433(9)$ & $0.723(2)$ & $0.051^{*}$ & \\
\hline $\mathrm{H} 3 \mathrm{~F}$ & $0.5103(18)$ & $0.4984(11)$ & $0.693(2)$ & $0.051^{*}$ & \\
\hline $\mathrm{O} 4$ & $0.47512(11)$ & $0.40611(6)$ & $0.96546(16)$ & $0.0291(3)$ & \\
\hline $\mathrm{H} 4 \mathrm{C}$ & $0.4079(14)$ & $0.3972(9)$ & $0.956(3)$ & $0.044 *$ & \\
\hline H4D & $0.5051(17)$ & $0.3935(9)$ & $0.883(2)$ & $0.044^{*}$ & \\
\hline $\mathrm{O} 5$ & $0.33269(10)$ & $0.51618(6)$ & $1.05567(15)$ & $0.0309(3)$ & \\
\hline $\mathrm{H} 5 \mathrm{~A}$ & 0.320545 & 0.510448 & 1.156884 & $0.046^{*}$ & \\
\hline H5B & 0.288625 & 0.490680 & 1.003402 & $0.046^{*}$ & \\
\hline $\mathrm{S} 1$ & $0.14894(3)$ & $0.39419(2)$ & $0.92733(4)$ & $0.02016(12)$ & \\
\hline O6 & $0.26060(10)$ & $0.36508(6)$ & $0.92041(16)$ & $0.0339(3)$ & \\
\hline $\mathrm{O} 7$ & $0.15935(10)$ & $0.46130(5)$ & $0.88353(15)$ & $0.0297(3)$ & \\
\hline $\mathrm{O} 8$ & $0.07525(11)$ & $0.36247(6)$ & 0.81077 (16) & $0.0341(3)$ & \\
\hline O9 & $0.10124(13)$ & $0.38801(6)$ & $1.08500(15)$ & $0.0408(4)$ & \\
\hline
\end{tabular}


Atomic displacement parameters $\left(\AA^{2}\right)$

\begin{tabular}{lllllll}
\hline & $U^{11}$ & $U^{22}$ & $U^{33}$ & $U^{12}$ & $U^{13}$ & $U^{23}$ \\
\hline Ni1 & $0.0238(2)$ & $0.0199(2)$ & $0.0160(2)$ & 0.000 & $-0.00128(15)$ & 0.000 \\
O1 & $0.0262(9)$ & $0.0235(9)$ & $0.0357(10)$ & 0.000 & $-0.0058(8)$ & 0.000 \\
O2 & $0.0465(11)$ & $0.0277(10)$ & $0.0203(9)$ & 0.000 & $-0.0002(8)$ & 0.000 \\
N1 & $0.0257(10)$ & $0.0280(10)$ & $0.0172(9)$ & 0.000 & $-0.0027(8)$ & 0.000 \\
N2 & $0.0326(8)$ & $0.0234(7)$ & $0.0313(8)$ & $0.0027(6)$ & $-0.0018(6)$ & $-0.0024(6)$ \\
N3 & $0.0275(11)$ & $0.0381(12)$ & $0.0335(12)$ & 0.000 & $0.0076(9)$ & 0.000 \\
C1 & $0.0396(10)$ & $0.0397(11)$ & $0.0214(9)$ & $-0.0057(8)$ & $0.0016(8)$ & $0.0098(8)$ \\
C2 & $0.0439(10)$ & $0.0254(9)$ & $0.0392(11)$ & $-0.0015(8)$ & $-0.0059(9)$ & $0.0115(8)$ \\
C3 & $0.0316(13)$ & $0.0423(15)$ & $0.0292(13)$ & 0.000 & $-0.0114(11)$ & 0.000 \\
C4 & $0.0252(16)$ & $0.046(2)$ & $0.044(2)$ & $0.0077(14)$ & $-0.0074(15)$ & $-0.0045(16)$ \\
Ni2 & $0.0226(2)$ & $0.0206(2)$ & $0.0185(2)$ & $-0.00029(15)$ & $-0.00085(15)$ & $-0.00050(15)$ \\
O3 & $0.0442(8)$ & $0.0364(7)$ & $0.0206(6)$ & $0.0102(6)$ & $-0.0007(6)$ & $0.0031(5)$ \\
O4 & $0.0297(6)$ & $0.0264(6)$ & $0.0312(7)$ & $-0.0037(5)$ & $0.0030(6)$ & $-0.0036(5)$ \\
O5 & $0.0258(6)$ & $0.0392(7)$ & $0.0279(6)$ & $-0.0029(5)$ & $0.0000(5)$ & $-0.0073(6)$ \\
S1 & $0.0224(2)$ & $0.0187(2)$ & $0.0193(2)$ & $-0.00018(14)$ & $-0.00065(14)$ & $0.00073(14)$ \\
O6 & $0.0255(6)$ & $0.0294(7)$ & $0.0468(8)$ & $0.0032(5)$ & $-0.0021(6)$ & $-0.0059(6)$ \\
O7 & $0.0369(7)$ & $0.0207(6)$ & $0.0316(7)$ & $-0.0039(5)$ & $-0.0083(5)$ & $0.0049(5)$ \\
O8 & $0.0378(7)$ & $0.0280(6)$ & $0.0364(7)$ & $-0.0079(5)$ & $-0.0130(6)$ & $0.0017(5)$ \\
O9 & $0.0594(9)$ & $0.0344(7)$ & $0.0284(7)$ & $0.0040(7)$ & $0.0168(6)$ & $0.0024(6)$ \\
& & & & & &
\end{tabular}

Geometric parameters $\left(A,{ }^{\circ}\right)$

\begin{tabular}{|c|c|c|c|}
\hline $\mathrm{Ni} 1-\mathrm{O} 1$ & $2.1395(18)$ & $\mathrm{C} 2-\mathrm{H} 2 \mathrm{D}$ & 0.9700 \\
\hline $\mathrm{Ni} 1-\mathrm{O} 2$ & $2.0940(19)$ & $\mathrm{C} 3-\mathrm{H} 3 \mathrm{C}$ & 0.9700 \\
\hline $\mathrm{Ni} 1-\mathrm{N} 1$ & $2.0640(19)$ & $\mathrm{C} 3-\mathrm{H} 3 \mathrm{C}^{\mathrm{i}}$ & 0.9700 \\
\hline $\mathrm{Ni} 1-\mathrm{N} 2$ & $2.1217(15)$ & $\mathrm{C} 3-\mathrm{H} 3 \mathrm{D}$ & 0.9700 \\
\hline $\mathrm{Ni} 1-\mathrm{N} 2^{\mathrm{i}}$ & $2.1217(15)$ & $\mathrm{C} 3-\mathrm{H} 3 \mathrm{D}^{\mathrm{i}}$ & 0.9700 \\
\hline $\mathrm{Ni} 1-\mathrm{N} 3$ & $2.069(2)$ & $\mathrm{C} 3-\mathrm{C} 4$ & $1.496(4)$ \\
\hline $\mathrm{O} 1-\mathrm{H} 1$ & $0.78(2)$ & $\mathrm{C} 4-\mathrm{H} 4 \mathrm{~A}$ & 0.9700 \\
\hline $\mathrm{O} 1-\mathrm{H} 1^{\mathrm{i}}$ & $0.78(2)$ & $\mathrm{C} 4-\mathrm{H} 4 \mathrm{~B}$ & 0.9700 \\
\hline $\mathrm{O} 2-\mathrm{H} 2$ & $0.81(2)$ & $\mathrm{Ni} 2-\mathrm{O} 3^{\mathrm{ii}}$ & 2.0678 \\
\hline $\mathrm{O} 2-\mathrm{H} 2^{\mathrm{i}}$ & $0.81(2)$ & $\mathrm{Ni} 2-\mathrm{O} 3$ & $2.0678(13)$ \\
\hline $\mathrm{N} 1-\mathrm{C} 1$ & $1.483(2)$ & $\mathrm{Ni} 2-\mathrm{O} 4^{\mathrm{ii}}$ & $2.0511(13)$ \\
\hline $\mathrm{N} 1-\mathrm{C}^{\mathrm{i}}$ & $1.483(2)$ & $\mathrm{Ni} 2-\mathrm{O} 4$ & $2.0511(13)$ \\
\hline $\mathrm{N} 1-\mathrm{C} 3$ & $1.488(3)$ & $\mathrm{Ni} 2-\mathrm{O} 5$ & $2.0739(12)$ \\
\hline $\mathrm{N} 2-\mathrm{H} 2 \mathrm{~A}$ & 0.8900 & $\mathrm{Ni} 2-\mathrm{O} 5^{\mathrm{ii}}$ & $2.0739(12)$ \\
\hline $\mathrm{N} 2-\mathrm{H} 2 \mathrm{~B}$ & 0.8900 & $\mathrm{O} 3-\mathrm{H} 3 \mathrm{E}$ & $0.808(15)$ \\
\hline $\mathrm{N} 2-\mathrm{C} 2$ & $1.481(2)$ & $\mathrm{O} 3-\mathrm{H} 3 \mathrm{~F}$ & $0.851(15)$ \\
\hline $\mathrm{N} 3-\mathrm{H} 3 \mathrm{~A}^{\mathrm{i}}$ & 0.8900 & $\mathrm{O} 4-\mathrm{H} 4 \mathrm{C}$ & $0.826(15)$ \\
\hline $\mathrm{N} 3-\mathrm{H} 3 \mathrm{~A}$ & 0.8900 & $\mathrm{O} 4-\mathrm{H} 4 \mathrm{D}$ & $0.830(15)$ \\
\hline N3-H3B & 0.8900 & $\mathrm{O} 5-\mathrm{H} 5 \mathrm{~A}$ & 0.8756 \\
\hline $\mathrm{N} 3-\mathrm{H} 3 \mathrm{~B}^{\mathrm{i}}$ & 0.8900 & $\mathrm{O} 5-\mathrm{H} 5 \mathrm{~B}$ & 0.8759 \\
\hline $\mathrm{N} 3-\mathrm{C} 4$ & $1.468(4)$ & $\mathrm{S} 1-\mathrm{O} 6$ & $1.4679(13)$ \\
\hline $\mathrm{C} 1-\mathrm{H} 1 \mathrm{~A}$ & 0.9700 & $\mathrm{~S} 1-\mathrm{O} 7$ & $1.4878(12)$ \\
\hline $\mathrm{C} 1-\mathrm{H} 1 \mathrm{~B}$ & 0.9700 & $\mathrm{~S} 1-\mathrm{O} 8$ & $1.4826(12)$ \\
\hline
\end{tabular}




\begin{tabular}{|c|c|c|c|}
\hline $\mathrm{C} 1-\mathrm{C} 2$ & $1.514(3)$ & $\mathrm{S} 1-\mathrm{O} 9$ & $1.4537(13)$ \\
\hline $\mathrm{C} 2-\mathrm{H} 2 \mathrm{C}$ & 0.9700 & & \\
\hline $\mathrm{O} 2-\mathrm{Ni1}-\mathrm{O} 1$ & $88.70(8)$ & $\mathrm{C} 1-\mathrm{C} 2-\mathrm{H} 2 \mathrm{D}$ & 109.8 \\
\hline $\mathrm{O} 2-\mathrm{Ni} 1-\mathrm{N} 2$ & $96.06(4)$ & $\mathrm{H} 2 \mathrm{C}-\mathrm{C} 2-\mathrm{H} 2 \mathrm{D}$ & 108.2 \\
\hline $\mathrm{O} 2-\mathrm{Ni} 1-\mathrm{N} 2^{\mathrm{i}}$ & $96.06(4)$ & $\mathrm{N} 1-\mathrm{C} 3-\mathrm{H} 3 \mathrm{C}^{\mathrm{i}}$ & $109.06(3)$ \\
\hline $\mathrm{N} 1-\mathrm{Ni1}-\mathrm{O} 1$ & $92.87(8)$ & $\mathrm{N} 1-\mathrm{C} 3-\mathrm{H} 3 \mathrm{C}$ & 109.1 \\
\hline $\mathrm{N} 1-\mathrm{Ni} 1-\mathrm{O} 2$ & $178.42(8)$ & $\mathrm{N} 1-\mathrm{C} 3-\mathrm{H} 3 \mathrm{D}^{\mathrm{i}}$ & $109.07(10)$ \\
\hline $\mathrm{N} 1-\mathrm{Ni} 1-\mathrm{N} 2^{\mathrm{i}}$ & $84.07(4)$ & $\mathrm{N} 1-\mathrm{C} 3-\mathrm{H} 3 \mathrm{D}$ & 109.1 \\
\hline $\mathrm{N} 1-\mathrm{Ni} 1-\mathrm{N} 2$ & $84.07(4)$ & $\mathrm{N} 1-\mathrm{C} 3-\mathrm{C} 4$ & $112.6(2)$ \\
\hline $\mathrm{N} 1-\mathrm{Ni1}-\mathrm{N} 3$ & $84.39(9)$ & $\mathrm{H} 3 \mathrm{C}-\mathrm{C} 3-\mathrm{H} 3 \mathrm{C}^{\mathrm{i}}$ & 134.6 \\
\hline $\mathrm{N} 2 \mathrm{i}-\mathrm{Ni} 1-\mathrm{O} 1$ & $85.52(4)$ & $\mathrm{H} 3 \mathrm{C}^{\mathrm{i}}-\mathrm{C} 3-\mathrm{H} 3 \mathrm{D}^{\mathrm{i}}$ & 107.8 \\
\hline $\mathrm{N} 2-\mathrm{Ni1}-\mathrm{O} 1$ & $85.52(4)$ & $\mathrm{H} 3 \mathrm{C}-\mathrm{C} 3-\mathrm{H} 3 \mathrm{D}$ & 107.8 \\
\hline $\mathrm{N} 2 \mathrm{i}-\mathrm{Ni} 1-\mathrm{N} 2$ & $164.74(9)$ & $\mathrm{H} 3 \mathrm{C}-\mathrm{C} 3-\mathrm{H} 3 \mathrm{D}^{\mathrm{i}}$ & 35.2 \\
\hline $\mathrm{N} 3-\mathrm{Ni} 1-\mathrm{O} 1$ & $177.27(8)$ & $\mathrm{H} 3 \mathrm{D}-\mathrm{C} 3-\mathrm{H} 3 \mathrm{C}^{\mathrm{i}}$ & 35.2 \\
\hline $\mathrm{N} 3-\mathrm{Ni} 1-\mathrm{O} 2$ & $94.03(9)$ & $\mathrm{H} 3 \mathrm{D}-\mathrm{C} 3-\mathrm{H} 3 \mathrm{D}^{\mathrm{i}}$ & 75.0 \\
\hline $\mathrm{N} 3-\mathrm{Ni} 1-\mathrm{N} 2^{\mathrm{i}}$ & $94.18(4)$ & $\mathrm{C} 4-\mathrm{C} 3-\mathrm{H} 3 \mathrm{C}$ & 109.1 \\
\hline $\mathrm{N} 3-\mathrm{Ni} 1-\mathrm{N} 2$ & $94.18(4)$ & $\mathrm{C} 4-\mathrm{C} 3-\mathrm{H} 3 \mathrm{C}^{\mathrm{i}}$ & $77.37(16)$ \\
\hline $\mathrm{Ni} 1-\mathrm{O} 1-\mathrm{H} 1^{\mathrm{i}}$ & $113.6(18)$ & $\mathrm{C} 4-\mathrm{C} 3-\mathrm{H} 3 \mathrm{D}^{\mathrm{i}}$ & $133.34(17)$ \\
\hline $\mathrm{Ni1}-\mathrm{O} 1-\mathrm{H} 1$ & $113.6(18)$ & $\mathrm{C} 4-\mathrm{C} 3-\mathrm{H} 3 \mathrm{D}$ & 109.1 \\
\hline $\mathrm{H} 1-\mathrm{O} 1-\mathrm{H} 1^{\mathrm{i}}$ & $109(3)$ & $\mathrm{N} 3-\mathrm{C} 4-\mathrm{H} 3 \mathrm{~A}^{\mathrm{i}}$ & $34.21(10)$ \\
\hline $\mathrm{Ni} 1-\mathrm{O} 2-\mathrm{H} 2$ & $111.3(19)$ & $\mathrm{N} 3-\mathrm{C} 4-\mathrm{C} 3$ & $113.2(3)$ \\
\hline $\mathrm{Ni} 1-\mathrm{O} 2-\mathrm{H} 2^{\mathrm{i}}$ & $111.3(19)$ & $\mathrm{N} 3-\mathrm{C} 4-\mathrm{H} 4 \mathrm{~A}$ & 108.9 \\
\hline $\mathrm{H} 2-\mathrm{O} 2-\mathrm{H} 2^{\mathrm{i}}$ & $103(4)$ & $\mathrm{N} 3-\mathrm{C} 4-\mathrm{H} 4 \mathrm{~B}$ & 108.9 \\
\hline $\mathrm{C} 1-\mathrm{i}-\mathrm{N} 1-\mathrm{Ni} 1$ & $104.58(11)$ & $\mathrm{C} 3-\mathrm{C} 4-\mathrm{H} 3 \mathrm{~A}^{\mathrm{i}}$ & $136.9(3)$ \\
\hline $\mathrm{C} 1-\mathrm{N} 1-\mathrm{Ni} 1$ & $104.58(11)$ & $\mathrm{C} 3-\mathrm{C} 4-\mathrm{H} 4 \mathrm{~A}$ & 108.9 \\
\hline $\mathrm{C} 1-\mathrm{N} 1-\mathrm{C} 1^{\mathrm{i}}$ & $113.0(2)$ & $\mathrm{C} 3-\mathrm{C} 4-\mathrm{H} 4 \mathrm{~B}$ & 108.9 \\
\hline $\mathrm{C} 1-\mathrm{N} 1-\mathrm{C} 3$ & $111.85(12)$ & $\mathrm{H} 4 \mathrm{~A}-\mathrm{C} 4-\mathrm{H} 3 \mathrm{~A}^{\mathrm{i}}$ & 76.7 \\
\hline $\mathrm{C} 1-\mathrm{i} 1-\mathrm{C} 3$ & $111.85(12)$ & $\mathrm{H} 4 \mathrm{~A}-\mathrm{C} 4-\mathrm{H} 4 \mathrm{~B}$ & 107.8 \\
\hline $\mathrm{C} 3-\mathrm{N} 1-\mathrm{Ni} 1$ & $110.50(15)$ & $\mathrm{H} 4 \mathrm{~B}-\mathrm{C} 4-\mathrm{H} 3 \mathrm{~A}^{\mathrm{i}}$ & 109.6 \\
\hline $\mathrm{Ni} 1-\mathrm{N} 2-\mathrm{H} 2 \mathrm{~A}$ & 110.0 & $\mathrm{O} 3{ }^{\mathrm{ii}}-\mathrm{Ni} 2-\mathrm{O} 3$ & 180.0 \\
\hline $\mathrm{Ni} 1-\mathrm{N} 2-\mathrm{H} 2 \mathrm{~B}$ & 110.0 & $\mathrm{O} 3^{\mathrm{ii}}-\mathrm{Ni} 2-\mathrm{O} 5$ & $89.88(5)$ \\
\hline $\mathrm{H} 2 \mathrm{~A}-\mathrm{N} 2-\mathrm{H} 2 \mathrm{~B}$ & 108.4 & $\mathrm{O} 3-\mathrm{Ni} 2-\mathrm{O} 5$ & $90.12(5)$ \\
\hline $\mathrm{C} 2-\mathrm{N} 2-\mathrm{Ni} 1$ & $108.29(11)$ & $\mathrm{O} 3^{\mathrm{ii}}-\mathrm{Ni} 2-\mathrm{O}^{\mathrm{ii}}$ & $90.12(5)$ \\
\hline $\mathrm{C} 2-\mathrm{N} 2-\mathrm{H} 2 \mathrm{~A}$ & 110.0 & $\mathrm{O} 3-\mathrm{Ni} 2-\mathrm{O}^{\mathrm{ii}}$ & $89.88(5)$ \\
\hline $\mathrm{C} 2-\mathrm{N} 2-\mathrm{H} 2 \mathrm{~B}$ & 110.0 & $\mathrm{O} 4-\mathrm{Ni} 2-\mathrm{O}^{\mathrm{ii}}$ & $92.87(5)$ \\
\hline $\mathrm{Ni} 1-\mathrm{N} 3-\mathrm{H} 3 \mathrm{~A}$ & 110.0 & $\mathrm{O} 4{ }^{\mathrm{ii}}-\mathrm{Ni} 2-\mathrm{O} 3$ & $92.87(5)$ \\
\hline $\mathrm{Ni1}-\mathrm{N} 3-\mathrm{H} 3 \mathrm{~A}^{\mathrm{i}}$ & $110.008(12)$ & $\mathrm{O} 4-\mathrm{Ni} 2-\mathrm{O} 3$ & $87.13(5)$ \\
\hline $\mathrm{Ni} 1-\mathrm{N} 3-\mathrm{H} 3 \mathrm{~B}^{\mathrm{i}}$ & $110.01(5)$ & $\mathrm{O} 4^{\mathrm{ii}}-\mathrm{Ni} 2-\mathrm{O} 3^{\mathrm{ii}}$ & $87.13(5)$ \\
\hline $\mathrm{Ni} 1-\mathrm{N} 3-\mathrm{H} 3 \mathrm{~B}$ & 110.0 & $\mathrm{O} 4 \mathrm{ii}-\mathrm{Ni} 2-\mathrm{O} 4$ & 180.0 \\
\hline $\mathrm{H} 3 \mathrm{~A}-\mathrm{N} 3-\mathrm{H} 3 \mathrm{~A}^{\mathrm{i}}$ & 133.8 & $\mathrm{O} 4^{\mathrm{ii}}-\mathrm{Ni} 2-\mathrm{O}^{\mathrm{ii}}$ & $93.28(5)$ \\
\hline $\mathrm{H} 3 \mathrm{~A}-\mathrm{N} 3-\mathrm{H} 3 \mathrm{~B}$ & 108.4 & $\mathrm{O} 4{ }^{\mathrm{ii}}-\mathrm{Ni} 2-\mathrm{O} 5$ & $86.72(5)$ \\
\hline $\mathrm{H} 3 \mathrm{~A}-\mathrm{N} 3-\mathrm{H} 3 \mathrm{~B}^{\mathrm{i}}$ & 34.7 & $\mathrm{O} 4-\mathrm{Ni} 2-\mathrm{O}^{\mathrm{ii}}$ & $86.72(5)$ \\
\hline $\mathrm{H} 3 \mathrm{~A}^{\mathrm{i}}-\mathrm{N} 3-\mathrm{H} 3 \mathrm{~B}^{\mathrm{i}}$ & 108.4 & $\mathrm{O} 4-\mathrm{Ni} 2-\mathrm{O} 5$ & $93.28(5)$ \\
\hline $\mathrm{H} 3 \mathrm{~B}-\mathrm{N} 3-\mathrm{H} 3 \mathrm{~A}^{\mathrm{i}}$ & 34.7 & $\mathrm{O} 5-\mathrm{Ni} 2-\mathrm{O}^{\mathrm{ii}}$ & $180.00(7)$ \\
\hline $\mathrm{H} 3 \mathrm{~B}-\mathrm{N} 3-\mathrm{H} 3 \mathrm{~B}^{\mathrm{i}}$ & 76.4 & $\mathrm{Ni} 2-\mathrm{O} 3-\mathrm{H} 3 \mathrm{E}$ & $124.9(15)$ \\
\hline $\mathrm{C} 4-\mathrm{N} 3-\mathrm{Ni} 1$ & $108.42(19)$ & $\mathrm{Ni} 2-\mathrm{O} 3-\mathrm{H} 3 \mathrm{~F}$ & $119.7(15)$ \\
\hline $\mathrm{C} 4-\mathrm{N} 3-\mathrm{H} 3 \mathrm{~A}$ & 110.0 & $\mathrm{H} 3 \mathrm{E}-\mathrm{O} 3-\mathrm{H} 3 \mathrm{~F}$ & $103(2)$ \\
\hline
\end{tabular}




\begin{tabular}{|c|c|c|c|c|}
\hline $\mathrm{C} 4-\mathrm{N} 3-\mathrm{H} 3 \mathrm{~A}^{\mathrm{i}}$ & $77.77(16)$ & $\mathrm{Ni} 2-\mathrm{O} 4-\mathrm{H} 4 \mathrm{C}$ & \multicolumn{2}{|c|}{$112.3(14)$} \\
\hline $\mathrm{C} 4-\mathrm{N} 3-\mathrm{H} 3 \mathrm{~B}$ & 110.0 & $\mathrm{Ni} 2-\mathrm{O} 4-\mathrm{H} 4 \mathrm{D}$ & \multicolumn{2}{|c|}{$112.1(14)$} \\
\hline $\mathrm{C} 4-\mathrm{N} 3-\mathrm{H} 3 \mathrm{~B}^{\mathrm{i}}$ & $\begin{array}{l}110.0 \\
135.62(17)\end{array}$ & $\mathrm{H} 4 \mathrm{C}-\mathrm{O} 4-\mathrm{H} 4 \mathrm{D}$ & \multicolumn{2}{|c|}{$105(2)$} \\
\hline $\mathrm{N} 1-\mathrm{C} 1-\mathrm{H} 1 \mathrm{~A}$ & 109.6 & $\mathrm{Ni} 2-\mathrm{O} 5-\mathrm{H} 5 \mathrm{~A}$ & \multicolumn{2}{|c|}{110.9} \\
\hline $\mathrm{N} 1-\mathrm{C} 1-\mathrm{H} 1 \mathrm{~B}$ & 109.6 & $\mathrm{Ni} 2-\mathrm{O} 5-\mathrm{H} 5 \mathrm{~B}$ & \multicolumn{2}{|c|}{110.8} \\
\hline $\mathrm{N} 1-\mathrm{C} 1-\mathrm{C} 2$ & $110.31(15)$ & $\mathrm{H} 5 \mathrm{~A}-\mathrm{O} 5-\mathrm{H} 5 \mathrm{~B}$ & \multicolumn{2}{|c|}{107.8} \\
\hline $\mathrm{H} 1 \mathrm{~A}-\mathrm{C} 1-\mathrm{H} 1 \mathrm{~B}$ & 108.1 & \multirow{2}{*}{\multicolumn{2}{|c|}{$\begin{array}{l}\mathrm{O} 6-\mathrm{S} 1-\mathrm{O} 7 \\
\mathrm{O} 6-\mathrm{S} 1-\mathrm{O} 8\end{array}$}} & \\
\hline $\mathrm{C} 2-\mathrm{C} 1-\mathrm{H} 1 \mathrm{~A}$ & 109.6 & & & $108.32(8)$ \\
\hline $\mathrm{C} 2-\mathrm{C} 1-\mathrm{H} 1 \mathrm{~B}$ & 109.6 & $\mathrm{O} 8-\mathrm{S} 1-\mathrm{O} 7$ & \multicolumn{2}{|c|}{$109.01(7)$} \\
\hline $\mathrm{N} 2-\mathrm{C} 2-\mathrm{C} 1$ & $109.38(14)$ & $\mathrm{O} 9-\mathrm{S} 1-\mathrm{O} 6$ & \multicolumn{2}{|c|}{$110.55(9)$} \\
\hline $\mathrm{N} 2-\mathrm{C} 2-\mathrm{H} 2 \mathrm{C}$ & 109.8 & $\mathrm{O} 9-\mathrm{S} 1-\mathrm{O} 7$ & \multicolumn{2}{|c|}{$110.37(8)$} \\
\hline $\mathrm{N} 2-\mathrm{C} 2-\mathrm{H} 2 \mathrm{D}$ & 109.8 & $\mathrm{O} 9-\mathrm{S} 1-\mathrm{O} 8$ & \multicolumn{2}{|c|}{$109.63(8)$} \\
\hline $\mathrm{C} 1-\mathrm{C} 2-\mathrm{H} 2 \mathrm{C}$ & 109.8 & & & \\
\hline $\mathrm{Ni} 1-\mathrm{N} 1-\mathrm{C} 1-\mathrm{C} 2$ & $-48.90(17)$ & $\mathrm{N} 1-\mathrm{C} 3-\mathrm{C} 4-\mathrm{N} 3$ & & $8(3)$ \\
\hline $\mathrm{Ni} 1-\mathrm{N} 1-\mathrm{C} 3-\mathrm{C} 4$ & $18.68(18)$ & $\mathrm{C} 1^{\mathrm{i}-\mathrm{N} 1}-\mathrm{C} 1-\mathrm{C} 2$ & & $.01(12)$ \\
\hline $\mathrm{Ni} 1-\mathrm{N} 2-\mathrm{C} 2-\mathrm{C} 1$ & $-27.22(18)$ & $\mathrm{C} 1-\mathrm{N} 1-\mathrm{C} 3-\mathrm{C} 4$ & & $4(2)$ \\
\hline $\mathrm{Ni} 1-\mathrm{N} 3-\mathrm{C} 4-\mathrm{C} 3$ & $34.2(3)$ & $\mathrm{C} 1-\mathrm{i} 1-\mathrm{C} 3-\mathrm{C} 4$ & & $7(2)$ \\
\hline $\mathrm{N} 1-\mathrm{C} 1-\mathrm{C} 2-\mathrm{N} 2$ & $52.2(2)$ & $\mathrm{C} 3-\mathrm{N} 1-\mathrm{C} 1-\mathrm{C} 2$ & & (2) \\
\hline Symmetry codes: (i) $x,-y$ & $-1,-z+2$ & & & \\
\hline Hydrogen-bond geon & & & & \\
\hline$D-\mathrm{H} \cdots A$ & $D-\mathrm{H}$ & $\mathrm{H} \cdots A$ & $D^{\cdots} A$ & $D-\mathrm{H} \cdots A$ \\
\hline $\mathrm{O} 1-\mathrm{H} 1 \cdots \mathrm{O} 8^{\mathrm{iii}}$ & $0.78(2)$ & $2.05(2)$ & $2.8212(16)$ & $172(2)$ \\
\hline $\mathrm{O} 2-\mathrm{H} 2 \cdots \mathrm{O} 6$ & $0.81(2)$ & $1.96(2)$ & $2.7342(15)$ & $162(3)$ \\
\hline $\mathrm{O} 3-\mathrm{H} 3 E^{\cdots} \cdot \mathrm{O}^{\text {iv }}$ & $0.81(2)$ & $1.94(2)$ & $2.731(2)$ & $167(2)$ \\
\hline $\mathrm{O} 3-\mathrm{H} 3 F^{\cdots} \cdots \mathrm{O} 7^{\mathrm{iii}}$ & $0.85(2)$ & $2.05(2)$ & $2.8403(18)$ & $155(2)$ \\
\hline $\mathrm{O} 4-\mathrm{H} 4 C \cdots \mathrm{O} 6$ & $0.83(2)$ & $1.91(2)$ & $2.7249(18)$ & $171(2)$ \\
\hline $\mathrm{O} 4-\mathrm{H} 4 D^{\cdots}{ }^{\mathrm{O}} 8^{\mathrm{iii}}$ & $0.83(2)$ & $1.95(2)$ & $2.7810(18)$ & $179(2)$ \\
\hline $\mathrm{O} 5-\mathrm{H} 5 A^{\cdots} \cdot \mathrm{O} 7^{\mathrm{v}}$ & 0.88 & 2.02 & $2.8125(19)$ & 150 \\
\hline $\mathrm{O} 5-\mathrm{H} 5 B^{\cdots} \cdots \mathrm{O} 7$ & 0.88 & 1.95 & $2.7826(17)$ & 160 \\
\hline
\end{tabular}

Symmetry codes: (iii) $x+1 / 2, y,-z+3 / 2$; (iv) $-x+1 / 2,-y+1, z-1 / 2$; (v) $-x+1 / 2,-y+1, z+1 / 2$. 